\title{
Controlling split-ring resonators with light
}

\author{
Polina V. Kapitanova, ${ }^{1}$ Stanislav I. Maslovski, ${ }^{1,2}$ Ilya V. Shadrivov, ${ }^{1,3, a)}$ Pavel M. Voroshilov, ${ }^{1}$ \\ Dmitry S. Filonov, ${ }^{1}$ Pavel A. Belov, ${ }^{1,4}$ and Yuri S. Kivshar ${ }^{1,3}$ \\ ${ }^{1}$ National Research University of Information Technologies, Mechanics and Optics, St. Petersburg 197101, \\ Russia \\ ${ }^{2}$ Instituto de Telecomunicações, Universidade de Coimbra, Pólo II, 3030-290 Coimbra, Portugal \\ ${ }^{3}$ Nonlinear Physics Centre, Research School of Physics and Engineering, Australian National University, \\ Canberra ACT 0200, Australia \\ ${ }^{4}$ Queen Mary University of London, Mile End Road, London E1 4NS, United Kingdom
}

(Received 14 November 2011; accepted 1 December 2011; published online 23 December 2011)

We propose an original approach for creating tunable electromagnetic metamaterials. We demonstrate experimentally that magnetic resonance of a split-ring resonator ("meta-atom" of a composite material) with a photodiode operated in photovoltaic mode can be tuned by changing the intensity of an external light source. Moreover, for two coupled resonators, we show that we can achieve light-induced switching between dark- and bright-mode responses. (C) 2011 American Institute of Physics. [doi:10.1063/1.3671617]

Electromagnetic metamaterials demonstrate many intriguing properties such as artificial magnetism at optical frequencies, negative refraction, enhanced chirality, and optical activity $^{1-3}$ (see also the recent review papers ${ }^{4,5}$ and references therein). Metamaterials can find many useful applications, e.g., for super-resolution and transformation optics. ${ }^{6,7}$ Majority of the demonstrated metamaterials possess fixed properties, but currently, many efforts are being made to achieve tunability of the metamaterial properties, either externally $^{8}$ or by employing their nonlinear response. ${ }^{9}$

At microwave frequencies, it is easy to design a tunable nonlinear meta-atom as a split-ring resonator (SRR) loaded with a varactor diode that operates in either bias-free or biased regimes ${ }^{10,11}$ and subsequently create a bulk nonlinear magnetic metamaterial ${ }^{12}$ allowing a power-induced control of the wave transmission. Moreover, such artificial nonlinear media allow the demonstration of many interesting phenomena accessible at low powers such as a nonlinear-optical mirror. ${ }^{13,14}$

In this Letter, we demonstrate an original concept for creating tunable metamaterials and make the first step towards creating a novel generation of metamaterials with the useful functionalities (e.g., lenses or waveguides) written or erased by light. We study experimentally SRRs with varactor diodes, in which biasing is supplied by photodiodes operated in the photovoltaic mode, and demonstrate that SRR's magnetic resonance can be tuned by changing the intensity of an external light source. We employ coupled SRRs to demonstrate enhancement of the resonant response and switching between the bright and dark modes.

In order to study the tunability of microwave metamaterials by external light, first, we consider a single lightsensitive SRR. The schematic of the SRR structure is shown in Fig. 1(a). The design of this tunable magnetic meta-atom is based on the standard side-coupled SRR formed by two broken rings of metal strip (copper) printed on a dielectric substrate (FR4 fiberglass, $\varepsilon_{\mathrm{r}} \approx 4.4$ ). To achieve tunability, we

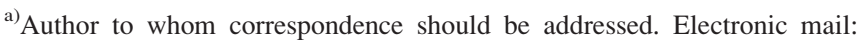
ivs124@physics.anu.edu.au.
}

introduce additional gap in the outer ring of the SRR in which we solder a varactor diode. The bias voltage for the varactor diode is produced by a photodiode that operates in the photovoltaic mode. To prevent shortening of the varactor by the large parasitic capacitance of the photodiode, we use chip inductors connected in series with the photodiode. An efficient blocking is achieved at the self-resonant frequency of the inductors that is chosen to be close to the operational frequency of the structure. As the voltage produced by the photodiode depends on the intensity of the incident light, the capacitance of the varactor changes with the light intensity. In turn, the resonant frequency of the SRR depends on the total loading capacitance per the entire loop, to which the capacitance of the varactor contributes. In this way, the resonant frequency of the SRR becomes dependent on the intensity of the light.

We use SRRs identical to those described in Ref. 10, with the radius of the inner ring of $2.56 \mathrm{~mm}$ and the metal strips width of $1.44 \mathrm{~mm}$, with the gap between the inner and outer rings of $0.32 \mathrm{~mm}$. The upper split in the outer ring and the symmetric split in the inner ring have the same width of $0.32 \mathrm{~mm}$. In our design, we mount a Skyworks ${ }^{\mathrm{TM}}$ SMV1233 varactor diode in the bottom split in the outer ring. Other elements (two chip inductors with the self-resonant frequency around $2.3 \mathrm{GHz}$ and a BPW-34-S photodiode from Opto Semiconductors ${ }^{\mathrm{TM}}$ ) are soldered to small metallic patches near the SRR that do not affect the magnetic resonance of the SRR.

To measure the magnetic response of the fabricated particle, one must excite the SRR with an external magnetic field. At decimeter wavelengths, this may be easily done with a small coupling loop (with the circumference much shorter than the wavelength) connected to a source of the high-frequency signal. However, this simple approach has drawbacks, because typically, the available sources have unbalanced (unipolar) outputs. When a magnetic loop is excited by such a source, the high-frequency current in the loop acquires a non-uniform component that radiates as an electric dipole that couples electrically to the structure under test. In practice, such unwanted coupling may be even 
(a)

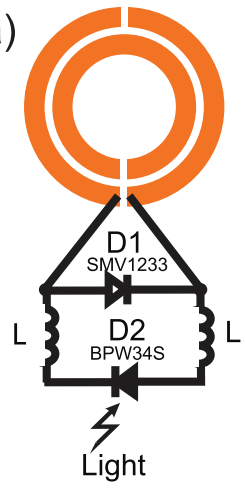

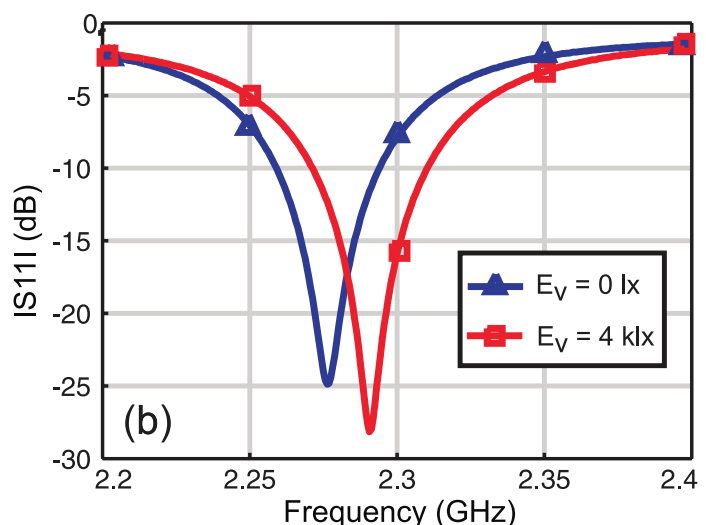

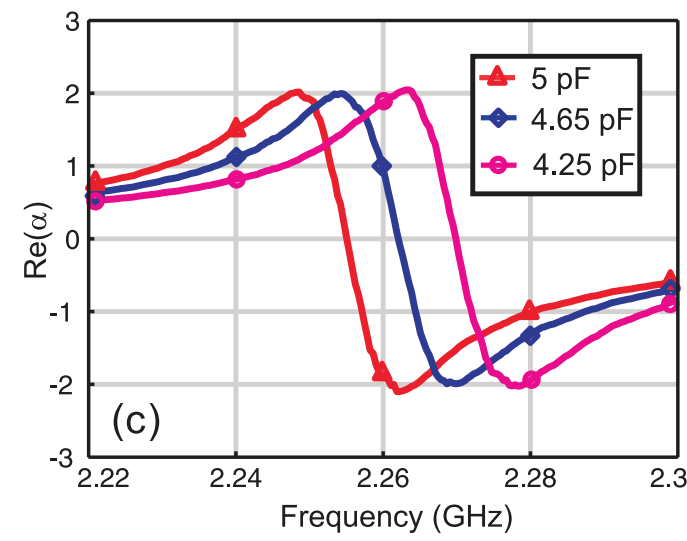

FIG. 1. (Color online) (a) Schematic of the light-tunable SRR and (b) measured reflection coefficient of tunable SRR with the resonance shifted by an external light with two values of illuminance $E_{v}$. (c) Real part of magnetic polarizability of the SRR extracted from simulations for different values of the capacitive load.

stronger than the required magnetic coupling, thus, affecting the measurement results dramatically.

To overcome this problem, specifically for the measurements of the magnetic response of the SRRs, we have designed a symmetric microstrip loop antenna. This antenna is fixed $5 \mathrm{~mm}$ above the plane of the SRR and connected to a vector network analyzer (VNA) using a coaxial cable. The reflection coefficient $S_{11}$ (in the cable) measured by the VNA when the loop antenna is coupled to a single tunable SRR is shown in Fig. 1(b) for different intensities of the incident light. In our experiments, we used photoluminescent lamp and controlled the light intensity near photodiode with lux meter. The minimum of the reflection coefficient corresponds to the maximum of the power that couples to the resonator which occurs at the resonance of the SRR. One may observe a shift in the resonant frequency of the SRR from 2.276 GHz to $2.29 \mathrm{GHz}$ when the light intensity at the photodiode increases from $01 \mathrm{x}$ to $4 \mathrm{klx}$. We note that the observed shift is on the order of the width of the resonance. Since this shift is caused by the change in the varactor capacitance, we can estimate this change with full-wave simulations of the SRR loaded with a bulk capacitor of a variable value. The results of these simulations are represented in Fig. 1(c), from which the change in the varactor capacitance may be identified as approximately $0.75 \mathrm{pF}$.

To improve the light sensitivity of single loaded SRRs, we have made several additional samples. In the first sample, the varactor diode is biased by two photodiodes connected in series in order to get higher voltage. In the second design, the SRR contains two back-to-back varactors which are biased by a single photodiode, each with the polarities of the bias voltages such that the capacitances of both varactors change equally. Both these designs provided $30 \mathrm{MHz}$ resonant frequency shift when the light intensity changed from 0 lx to $4 \mathrm{klx}$.

The sensitivity of the studied meta-atoms to the external light may be also increased using a qualitatively different approach. Instead of considering meta-atoms formed by single SRRs, one may consider structures composed of several coupled resonators. When two or more identical resonant structures interact with each other, the resonances of the coupled system split into a set of frequencies that correspond to various distributions of the amplitudes and the phases of the oscillations among the resonators. Such independent collective oscillations are known as normal modes.

In the case of two coupled SRRs (we place the SRRs in parallel planes with their axes coinciding, see Fig. 2(a)), there exist two normal modes: one has currents in both SRRs circulating in the same direction (even mode) and another has currents circulating in the opposite direction (odd mode). When such a pair is placed into a uniform external magnetic field, the even mode couples strongly to the external field, while the odd mode is not excited at all. Therefore, the even mode is called bright mode, and it is associated with strong total magnetic moment, strong scattering, and a lower frequency, and the odd mode is called dark mode, which has vanishing magnetic moment and scattering, and a higher frequency. The splitting of the resonant frequencies of these modes is controlled by the strength of coupling that can be adjusted by changing the separation between the two
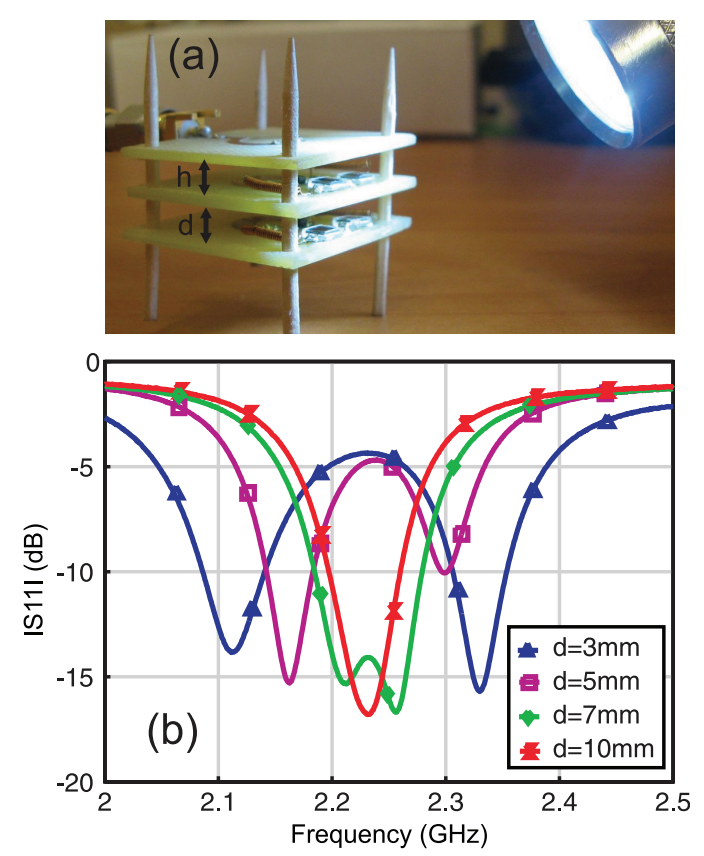

FIG. 2. (Color online) (a) Photograph of tunable SRRs (two lower boards) excited by a symmetric loop antenna (top board) and (b) measured reflection coefficient for different distances between SRRs and illuminance 7001x (sample with two photodiodes). 

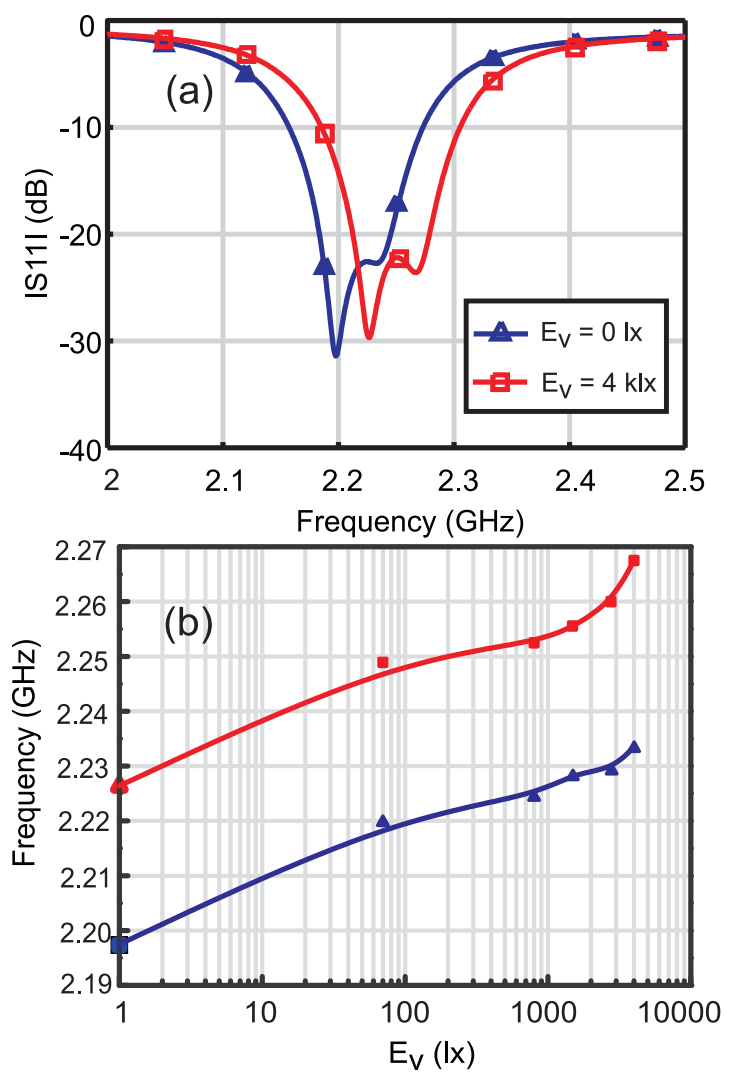

FIG. 3. (Color online) (a) Switching between the dark and bright mode and (b) dependence of the resonant frequencies of odd and even modes as a function of light intensity $E_{v}$.

interacting SRRs. Here, we fine-tune the coupling and, respectively, the splitting of the resonant frequencies in the pair of SRRs without applied light so that it equals to the shift in the resonant frequency of an isolated SRR when the intensity of light is changed. As a result, in such structure, by changing the light intensity, it becomes possible to switch between the bright and dark modes for the fixed frequency of the electromagnetic wave. When such a pair of resonators is placed in a uniform field (for example, to the field of an incident plane wave with the wavelength much larger than the characteristic size of the meta-atom), the SRR pair will have a much better tunability by light, because the effective magnetization of such a pair will change dramatically when the light intensity changes.

To check this idea, we perform a series of experiments with mutually coupled tunable SRRs [see Fig. 2(a)]. The same balanced magnetic loop antenna is used to measure the response of the structure. The antenna is placed at the fixed distance $h=5 \mathrm{~mm}$ above the upper SRR. First, we studied the splitting of the resonant frequencies of even and odd modes (note that both bright and dark modes can be excited in this setup, because the external magnetic field is not uniform along the axis of the structure). The distance $d$ between the upper and lower SRR was adjusted during the measurements. The measurements were done with the photodiode illuminated by light with the intensity of $700 \mathrm{~lx}$. Although no special efforts were made to provide equal illumination of the photodiodes in the top and bottom SRRs, the obtained results plotted in Fig. 2(b) demonstrate that the mean frequency of all the curves stays approximately the same when the distance between the SRRs changes. As expected, when the distance between two SRRs is too large, the coupling is too weak, and only a single resonant peak is observed. When the separation is reduced, the splitting increases.

Finally, Fig. 3(a) demonstrates the light-induced switching between the bright and dark modes for the case when the SRRs are separated by $d=8 \mathrm{~mm}$. When the illumination strength is changed from $0 \mathrm{~lx}$ to $4 \mathrm{klx}$, the resonant curve shifts to the right. As a result, if the external electromagnetic wave with the frequency around $2.23 \mathrm{GHz}$ interacts with the dark mode when the photodiode is not illuminated by light, then by switching the light on, the properties of the coupled SRRs change such that the electromagnetic wave excites the bright mode. In other words, initially nonmagnetic metaparticle will acquire a significant magnetic response. Finally, the dependence of the resonant frequencies of these modes on the light intensity is presented in Fig. 3(b).

In conclusion, we have suggested and demonstrated experimentally an original approach for tunability of metaatoms, the key elements of electromagnetic metamaterials. We have fabricated SRRs loaded with photodiodes and demonstrated a shift of the magnetic resonance by an external light source. We have employed several approaches of collective SRR response to enhance this tunability. We believe that this approach can be useful for creating bulk metamaterials whose properties can be controlled by light. Importantly, by illuminating individual meta-atoms with light of varying intensities, it will be possible to produce dynamically reconfigurable spatially inhomogeneous structures such as graded index lenses, prisms, mirrors, etc.

The authors acknowledge a support from the Ministry of Education and Science of the Russian Federation, Dynasty Foundation (Russia), the Engineering and Physical Sciences Research Council (UK), and the Australian Research Council (Australia).

\footnotetext{
${ }^{1}$ V. G. Veselago, Sov. Phys. Usp. 8, 2854 (1967) [Sov. Phys. Usp. 10, 509 (1968)].

${ }^{2}$ J. B. Pendry, Phys. Rev. Lett. 85, 3966 (2000).

${ }^{3}$ R. A. Shelby, D. R. Smith, and S. Schultz, Science 292, 77 (2001).

${ }^{4}$ C. M. Soukoulis and M. Wegener, Science 330, 1633 (2010).

${ }^{5}$ A. D. Boardman, V. V. Grimalsky, Yu. S. Kivshar, S. V. Koshevaya, M. Lapine, N. M. Litchinitser, V. N. Malnev, M. Noginov, Yu. G. Rapoport, and V. M. Shalaev, Laser Photonics Rev. 5, 287 (2011).

${ }^{6}$ U. Leonhardt, Science 312, 1777 (2006).

${ }^{7}$ J. B. Pendry, D. Schurig, and D. R. Smith, Science 312, 1780 (2006).

${ }^{8}$ W. J. Padilla, A. J. Taylor, C. Highstrete, M. Lee, and R. D. Averitt, Phys. Rev. Lett. 96, 107401 (1996).

${ }^{9}$ A. A. Zharov, I. V. Shadrivov, and Yu. S. Kivshar, Phys. Rev. Lett. 91, 037401 (2003).

${ }^{10}$ I. V. Shadrivov, S. K. Morrison, and Yu. S. Kivshar, Opt. Express 14, 9344 (2006).

${ }^{11}$ B. Wang, J. Zhou, T. Koschny, and C. M. Soukoulis, Opt. Express 16, 16058 (2008).

${ }^{12}$ I. V. Shadrivov, A. B. Kozyrev, D. W. Van Der Werde, and Yu. S. Kivshar, Opt. Express 16, 20266 (2008).

${ }^{13}$ I. V. Shadrivov, A. A. Zharov, and Y. S. Kivshar, J. Opt. Soc. Am. B 23, 529 (2006).

${ }^{14}$ A. Rose, D. Huang, and D. R. Smith, Phys. Rev. Lett. 107, 063902 (2011).
} 